\title{
Estudo da intercalação de compostos orgânicos em caulins na forma ácida
}

\section{(Study of intercalation of organic compounds into kaolin in acid form)}

\author{
M. V. da S. Fernandes, L. R. D. da Silva* \\ Universidade Federal do Ceará, Av. Prof. Aurélio Câmara s/n, C.P. 6002, Campus do Pici, Fortaleza CE \\ 60451-970 pillaredclay@yahoo.com.br,lindomar@dqoi.ufc.br
}

\begin{abstract}
Resumo
A viabilidade da utilização de importantes argilas tais como os caulins em processos de adsorção, pode ocorrer mediante a modificação química através de intercalação em suas estruturas filitosas. Amostras de caulin da região do nordeste do Brasil, um branco e outro cinza de uma porção da região borborema-seridó no estado da Paraíba foram intercalados com dimetilsulfóxido, ácido oxálico e ácido cítrico. Na caracterização das amostras naturais e modificadas foram utilizadas técnicas de difração de raios $\mathrm{X}$, análise térmica e espectrometria de absorção na região do infravermelho.
\end{abstract}

Palavras-chave: caulim, intercalação, dimetil sulfóxido, ácido oxálico, ácido cítrico.

\section{Abstract}

The feasibility of using major clays such as kaolin in the adsorption processes, can occur through chemical modification by intercalation in their structures phyllite. Samples of kaolin in the region of northeast Brazil, one white and one gray of a portion of the region Borborema-served in the state of Paraiba, were interspersed with dimethylsulfoxide, oxalic acid and citric acid. The characterization natural and modified techniques were used X-ray diffraction, thermal analysis and absorption spectroscopy in the infrared.

Keywords: kaolin, intercalation, dimethyl sulfoxide, oxalic acid, citric acid.

\section{INTRODUÇ̃̃O}

Argilas são silicatos de $\mathrm{Al}, \mathrm{Fe}$ e $\mathrm{Mg}$ hidratados, com estruturas cristalinas em camadas (filossilicatos), constituídos por folhas contínuas de tetraedros $\mathrm{SiO}_{4}$, ordenados de forma hexagonal, condensados com folhas octaédricas de hidróxidos de metais tri e divalentes; a maioria das argilas são constituídas essencialmente por partículas (cristais) com dimensões geralmente abaixo de $2 \mu \mathrm{m}$ [1]. A caulinita pertencente ao grupo dos caulins que possuem estrutura disposta em empilhamento $1: 1$, onde as folhas de unidades tetraédricas de silício compartilham de um plano comum de átomos de oxigênio com a superfície octaédrica do alumínio. As camadas são mantidas juntas por um vértice em comum através de ligações hidrogênio entre os oxigênios dos tetraedros e as hidroxilas dos octaedros de alumínio [2,3]. Alguns autores afirmam que a capacidade de adsorção das argilas poderia ser melhorada através de modificações específicas baseadas em processos de intercalação com agentes orgânicos [4]. A alta área superficial, estabilidade química e mecânica, alta capacidade de troca catiônica (CTC), acidez de Brönsted e Lewis, entre outras, têm feito das argilas excelentes materiais de adsorção [5]. Os processos de adsorção são de grande importância na remoção de poluentes e mais recentemente para separação de gases [6].<smiles>CS(C)=O</smiles>

Dimetil Sulfóxido [7]<smiles>O=C(O)C(=O)O</smiles>

Ácido Oxálico [8]<smiles>O=C(O)CC(O)(CC(=O)O)C(=O)O</smiles>

Ácido Cítrico $[8,9]$
A intercalação de caulins na forma ácida com dimetilsulfóxido, ácido oxálico e ácido cítrico, após a remoção da matéria orgânica (ácido húmico e ácido fúlvico) e obtenção da forma amoniacal, tem como objetivo torná-los adsorventes de metais mais eficientes do que na sua forma pura. No entanto, a presente investigação visa essencialmente, o estudo da capacidade de intercalação dos compostos orgânicos mencionados em argilas cauliníticas na sua forma ácida.

\section{MATERIAIS E MÉTODOS}

Dois caulins foram utilizados, branco e cinza, ambos provenientes da região Borborema-Seridó no estado da Paraíba. Ambos foram secos em estufa a aproximadamente $90{ }^{\circ} \mathrm{C} / 24 \mathrm{~h}$, depois foram macerados e logo após foram peneirados em peneira de 200 mesh $(<74 \mu \mathrm{m})$ e em seguida foram armazenados em dessecador. Todas as soluções 
utilizadas foram preparadas com água deionizada. As soluções estoques foram preparadas a partir de quantidades apropriadas de cada reagente, como $\mathrm{NaCl} 1$ mol.L-1, hipoclorito de sódio 0,1 mol.L $\mathrm{L}^{-1}(\mathrm{pH}=9,5)$, solução tampão a $2 \%$ de $\mathrm{Na}_{2} \mathrm{CO}_{3}-\mathrm{NaHCO}_{3}$, acetato de amônio P.A. Vetec, ácido oxálico P.A.. Os reagentes dimetilsulfóxido (DMSO) P.A. Vetec com $\mathrm{d}=1,092$ g. $\mathrm{mL}^{-1}$ a $25{ }^{\circ} \mathrm{C}$, álcool metílico Synth, álcool etílico absoluto Synth com 99,5 vol\%, hidróxido de amônio P.A. foram utilizados na sua forma pura. A remoção da matéria orgânica foi realizada com $\mathrm{NaOCl} 0,1 \mathrm{~mol} . \mathrm{L}^{-1}$ a $10 \% \mathrm{~m} / \mathrm{v}$, sob agitação por $6 \mathrm{~h}(\mathrm{em}$ triplicata). Em seguida efetuou-se lavagem dos resíduos com solução tampão $\mathrm{Na}_{2} \mathrm{CO}_{3}-\mathrm{NaHCO}_{3}$ a $2 \%(\mathrm{pH}=9,5)$, sendo que cada lavagem consiste em adicionar $200 \mathrm{~mL}$ do tampão, agitar por $6 \mathrm{~h}$, centrifugar e separar o sobrenadante (em triplicata) e posteriormente lavar com água deionizada por no mínimo 5 vezes.

Massas de $1 \mathrm{~g}$ de caulim com matéria orgânica removida e na forma sódica foram misturadas com $20 \mathrm{~mL}$ de acetato de amônio 1 mol.L $\mathrm{L}^{-1}$ (em triplicata), colocadas em erlenmeyers de $250 \mathrm{~mL}$, agitando-se por $2 \mathrm{~h}$ em agitador orbital e em seguida lavando-se com etanol $96 \%$. Este procedimento resultou na forma amoniacal dos caulins, sendo que estes, posteriormente, foram colocados em cadinho de porcelana e em seguida levados a mufla a $300{ }^{\circ} \mathrm{C} / 2 \mathrm{~h}$, resultando na forma ácida. Foi pesado $1 \mathrm{~g}$ de ambos os caulins, colocandoos em reatores de teflon-alumínio, em seguida adicionouse $10 \mathrm{~mL}$ de DMSO ao reator, que foi fechado e deixado em estufa a $150{ }^{\circ} \mathrm{C} / 2 \mathrm{~h}$, seguido de filtração em papel de filtro quantitativo e lavado com $10 \mathrm{~mL}$ de metanol para retirar o excesso de DMSO e centrifugando-se por $20 \mathrm{~min}$ a $4000 \mathrm{rpm}$ (em duplicata), deixando-se secar por $24 \mathrm{~h}$ em capela sob exaustão. Em erlenmeyers de $250 \mathrm{~mL}$ foram adicionados $1 \mathrm{~g}$ de caulins na forma ácida, $50 \mathrm{~mL}$ de ácido oxálico $0,128 \mathrm{~mol} . \mathrm{L}^{-1}$, deixando-se sob agitação orbital por $2 \mathrm{~h}$ e lavando-se com $30 \mathrm{~mL}$ de metanol (em triplicata). O mesmo procedimento anteriormente citado também foi realizado com ácido cítrico.

Os experimentos de difração de raios $\mathrm{X}$ foram realizados em um difratômetro de pó com geometria Bragg-Brentano em modo contínuo com velocidade de $1 \%$ min, radiação Cuk $\alpha(\lambda=0,1542 \mathrm{~nm})$ com tubo a $40 \mathrm{kV}$ e $25 \mathrm{~mA}$.

As análises termogravimétricas foram realizadas em equipamento Shimadzu TGA-50H com amostras de massa $10 \mathrm{mg}$ e submetidas a aquecimento com razão de $10^{\circ} \mathrm{C} \cdot \mathrm{min}^{-1}$ até uma temperatura de decomposição de $400^{\circ} \mathrm{C}$ em cadinho de alumina SOB atmosfera de nitrogênio [10].

Os espectros na região do infravermelho foram na faixa $4000-400 \mathrm{~cm}^{-1}$ foram obtidos em um equipamento Shimadzu FTIR-8300 com material seco a $90{ }^{\circ} \mathrm{C} / 2 \mathrm{~h} \mathrm{em}$ estufa e posteriormente sob vácuo. As análises foram feitas em pastilhas de $\mathrm{KBr}$ na proporção de $3 \%(\mathrm{~m} / \mathrm{m})$ prensadas sob 3 ton.

\section{RESULTADOS E DISCUSSÃO}

De acordo com o difratograma A (Fig. 1), observa- se o pico $\mathrm{d}(001)$ referente à caulinita na forma ácida, que apresenta valor de $12,36^{\circ} 2 \theta$, correspondente a uma distância interplanar de $0,715 \mathrm{~nm}$, sendo característico de estrutura caulinítica [11]. No difratograma B observa-se que após o procedimento de intercalação com DMSO o pico d(001) da caulinita torna-se mais intenso, sendo deslocado para $7,89^{\circ} 2 \theta(1,121 \mathrm{~nm})$, em conseqüência de uma expansão da distância interplanar basal na ordem de 56,78\% (equação A):

$$
\% \text { Expansão }=\left[\left(\mathrm{d}_{2}-\mathrm{d}_{1}\right) / \mathrm{d}_{1}\right] \times 100
$$

na qual $d_{1}$ e d são as distâncias interplanares relacionadas ao pico $\mathrm{d}(001)$ da caulinita não-intercalada e intercalada, respectivamente. Os difratogramas $\mathrm{C}$ e D (Fig. 1) mostram que não ocorreu deslocamento do pico $\mathrm{d}(001)$ da caulinita, evidenciando assim que a modificação pode ter gerado um material apenas impregnado. No difratograma A (Fig. 2), observa-se o pico d(001) característico da caulinita com valor de $12,26^{\circ} 2 \theta$ correspondente a uma distância interplanar de $0,718 \mathrm{~nm}$ e possíveis traços de mica com $\mathrm{d}(001)$ em $8,84^{\circ}$ $2 \theta(0,999 \mathrm{~nm})$, enquanto que no difratograma B houve deslocamento do pico $\mathrm{d}(001)$ da caulinita para $7,89^{\circ} 2 \theta$ $(1,121 \mathrm{~nm})$, e o pico d(001), possivelmente da mica, não teve deslocamento. Observa-se também um pico remanescente em $12,32^{\circ} 2 \theta$, que pode estar relacionado a frações de caulim não intercaladas com DMSO.

Nas curvas TG/DTG do caulim branco ácido modificado com DMSO (Fig. 3) observa-se um evento em $182{ }^{\circ} \mathrm{C}$ referente à volatilização do DMSO, com perda de massa de $11 \%$, e para o caulim branco ácido modificado com ácido

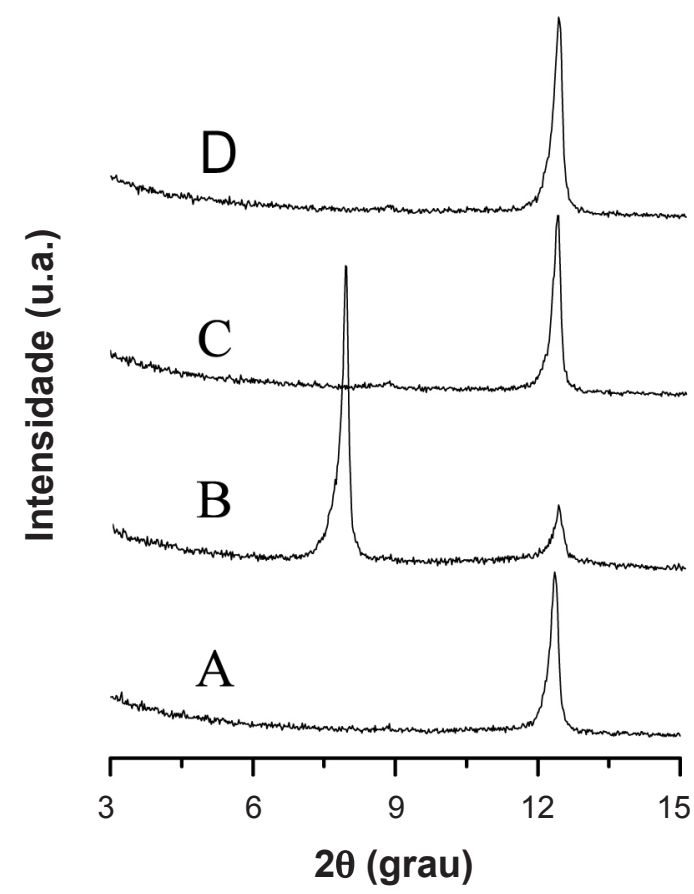

Figura 1: Difratogramas de raios $\mathrm{X}$ do caulim branco: A) ácido; B) ácido/DMSO; C) ácido/ácido oxálico e D) ácido/ácido cítrico. [Figure 1: XRD patterns of white kaolin : A) acid, B) acid / DMSO, C) acid / oxalic acid and D) acid/citric acid.] 


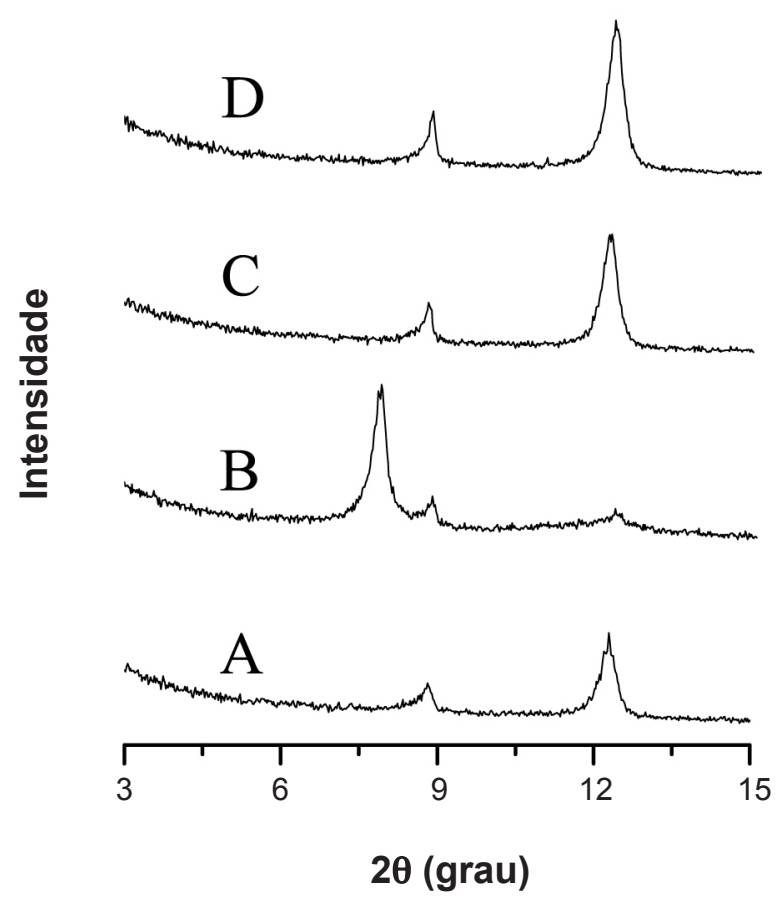

Figura 2: Difratogramas de raios X do caulim cinza: A) ácido; B) ácido/DMSO; C) ácido/ácido oxálico e D) ácido/ácido cítrico.

[Figure 2: XRD patterns of gray kaolin: A) acid, B) acid / DMSO, C) acid / oxalic acid and D) acid / citric acid.]

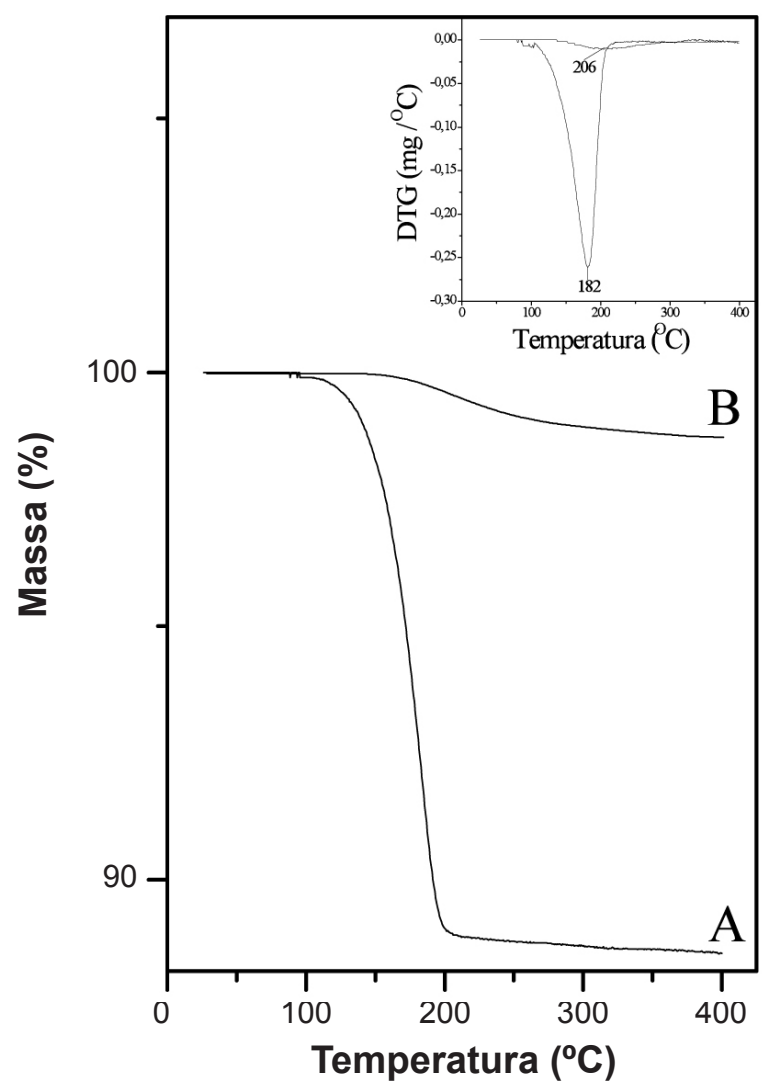

Figura 3: Curvas TG e DTG do caulim branco sob atmosfera de $\mathrm{N}_{2}$ a $10^{\circ} \mathrm{C} / \mathrm{min}:$ A) ácido/DMSO e B) ácido/ácido cítrico.

[Figure 3: TG and DTG curves of white kaolin under $\mathrm{N}_{2}$ at $10^{\circ} \mathrm{C}$ / min: A) acid / DMSO and B) acid / citric acid.] cítrico, um evento em $206^{\circ} \mathrm{C}$ com perda de massa de $1,3 \%$.

Nas curvas TG/DTG do caulim cinza ácido com DMSO (Fig. 4), observa-se um evento em $169^{\circ} \mathrm{C}$ referente à perda de massa de 7,3\% relacionada à saída do DMSO. Em $214{ }^{\circ} \mathrm{C}$ também ocorre perda de massa com uma variação de $~ 1,5 \%$, que tem sido atribuída ao ácido cítrico. Os resultados das curvas TG/DTG dos caulins modificados com ácido oxálico não apresentaram resultados expressivos. Uma análise comparativa da termogravimetria entre as espécies orgânicas utilizadas indicou que o DMSO interage em quantidades mássicas relativas bem maiores que os demais. Presumese que tal comportamento possa estar relacionado com a maior afinidade da estrutura do caulim por grupos sulfonilas do DMSO. Isto poderá ser melhor avaliado através da espectroscopia na região do infravermelho.

$\mathrm{O}$ resultado das análises de calorimetria diferencial exploratória (DSC) do caulim branco (Fig. 5), da forma ácida com DMSO (curva B), mostra a ocorrência de um evento endotérmico em $68^{\circ} \mathrm{C}$ e outro em $217^{\circ} \mathrm{C}$. Embora a termogravimetria tenha apresentado apenas um evento em $182{ }^{\circ} \mathrm{C}$, este valor de temperatura para a perda de massa verificada se deve à eliminação do DMSO sem que tenha havido sua degradação. O fato da temperatura na DSC ser maior que a da termogravimetria para o mesmo evento estaria associado à natureza da atmosfera utilizada uma vez

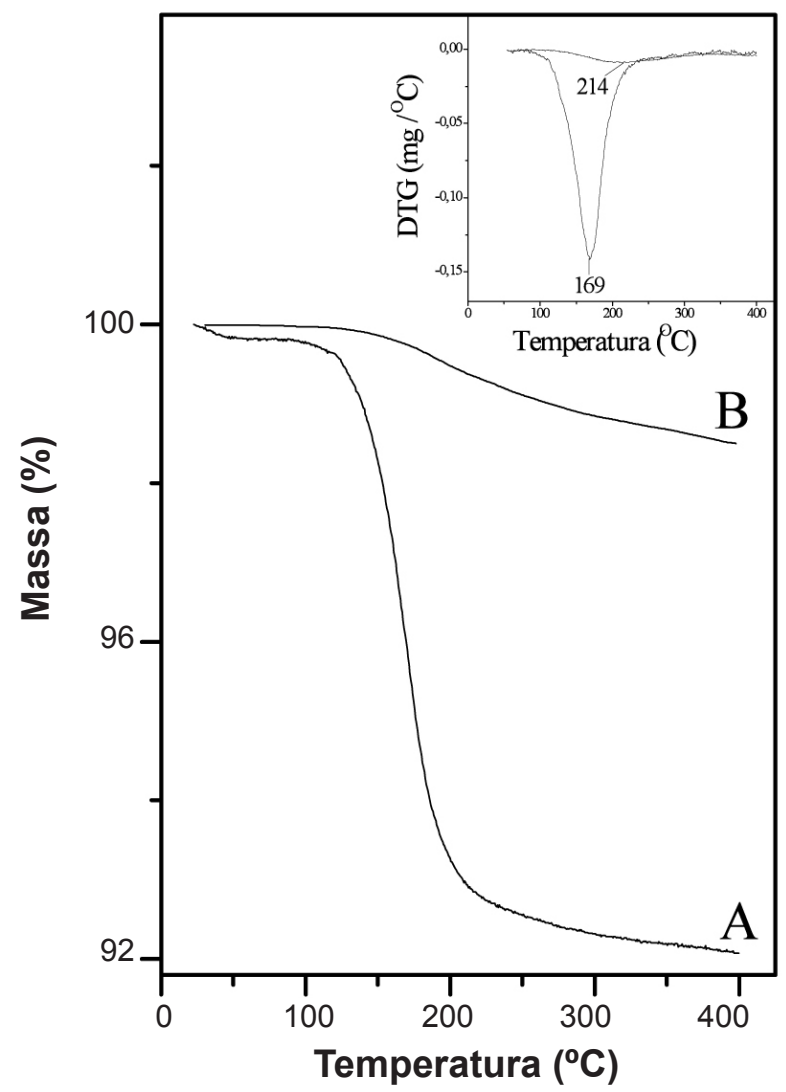

Figura 4: Curvas TG e DTG do caulim cinza sob $\mathrm{N}_{2}$ a $10{ }^{\circ} \mathrm{C} / \mathrm{min}$ : A) ácido/DMSO e B) ácido/ácido cítrico.

[Figure 4: TG and DTG of grey kaolin under $\mathrm{N}_{2}$ at $10^{\circ} \mathrm{C} / \mathrm{min}: \mathrm{A}$ ) acid / DMSO and B) acid / citric acid.] 


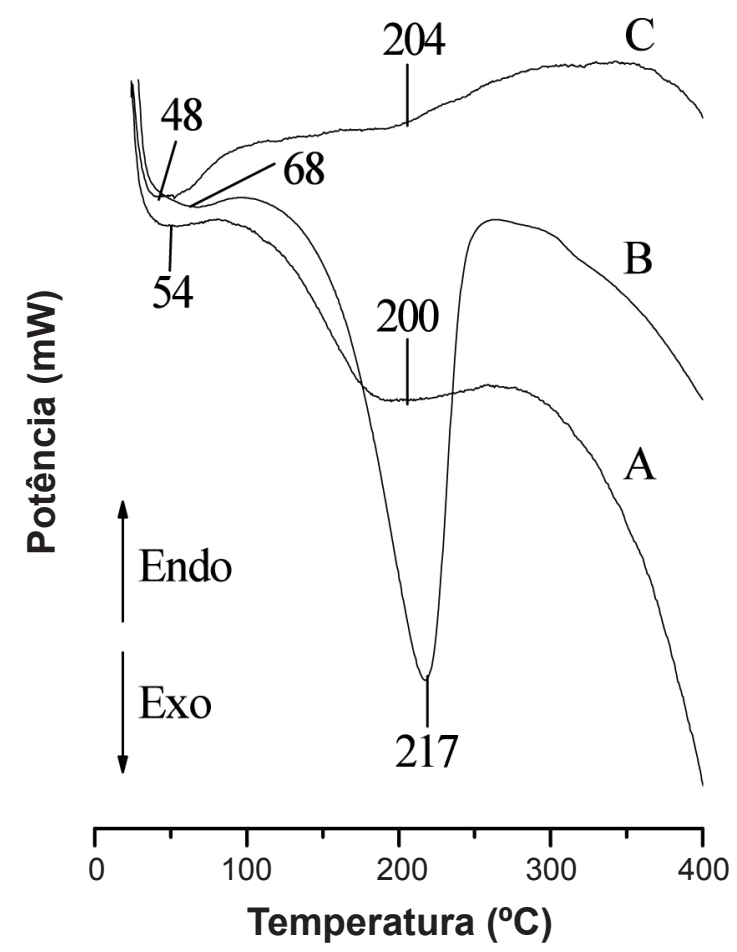

Figura 5: Curvas de calorimetria exploratória diferencial das formas modificadas de caulim branco sob $\mathrm{N}_{2}$ a $10{ }^{\circ} \mathrm{C} / \mathrm{min}$ : A) ácido/ ácido cítrico; B) ácido/DMSO e C) ácido/ácido oxálico.

[Figure 5: Differential scanning calorimetry curves of modified forms of white kaolin, with a heating rate of $10{ }^{\circ} \mathrm{C} / \mathrm{min}$ under $\mathrm{N}_{2}$ : A) acid / citric acid, B) acid / DMSO and C) acid / oxalic acid.]

que em atmosferas não oxidantes os fenômenos térmicos ocorrem em temperaturas mais elevadas [2]. O evento em $68{ }^{\circ} \mathrm{C}$ se deve à perda de umidade, e não se verificou qualquer interferência desta na eliminação do DMSO. O uso de atmosfera de nitrogênio se deve a uma limitação do equipamento.

Para o caulim branco/ácido cítrico (Fig. 5A) também ocorrem dois eventos em $54{ }^{\circ} \mathrm{C}$ e $200{ }^{\circ} \mathrm{C}$, ambos endotérmicos. Já para a forma modificada com ácido oxálico (Fig. 5C) há dois eventos endotérmicos, um em $48^{\circ} \mathrm{C}$ e outro em $204{ }^{\circ} \mathrm{C}$. Observa-se na curva B do caulim cinza (Fig. 6) dois eventos endotérmicos em $52{ }^{\circ} \mathrm{C}$ e em $196{ }^{\circ} \mathrm{C}$, enquanto que na curva $\mathrm{C}$ da mesma figura verifica-se a ocorrência de dois eventos endotérmicos em $54{ }^{\circ} \mathrm{C}$ e $220^{\circ} \mathrm{C}$ para o caulim cinza/ácido oxálico. Ocorrem dois eventos endotérmicos (Fig. 6A) para o caulim cinza em $78{ }^{\circ} \mathrm{C}$ e $211^{\circ} \mathrm{C}$. Os eventos são coincidentes com os resultados de perda de massa observados nas análises termogravimétricas. Portanto há uma tendência à saída do DMSO sem que haja sua degradação. Como esta característica se estende a todas as amostras com DMSO, acredita-se que pelo fato das energias envolvidas nas interações serem relativamente baixas, ocorram interações de natureza essencialmente física. E não é clara a participação dos sítios ácidos nos processos de interação entre estrutura DMSO, principalmente devido a ausência de evidência de degradação induzida por sítios ácidos.

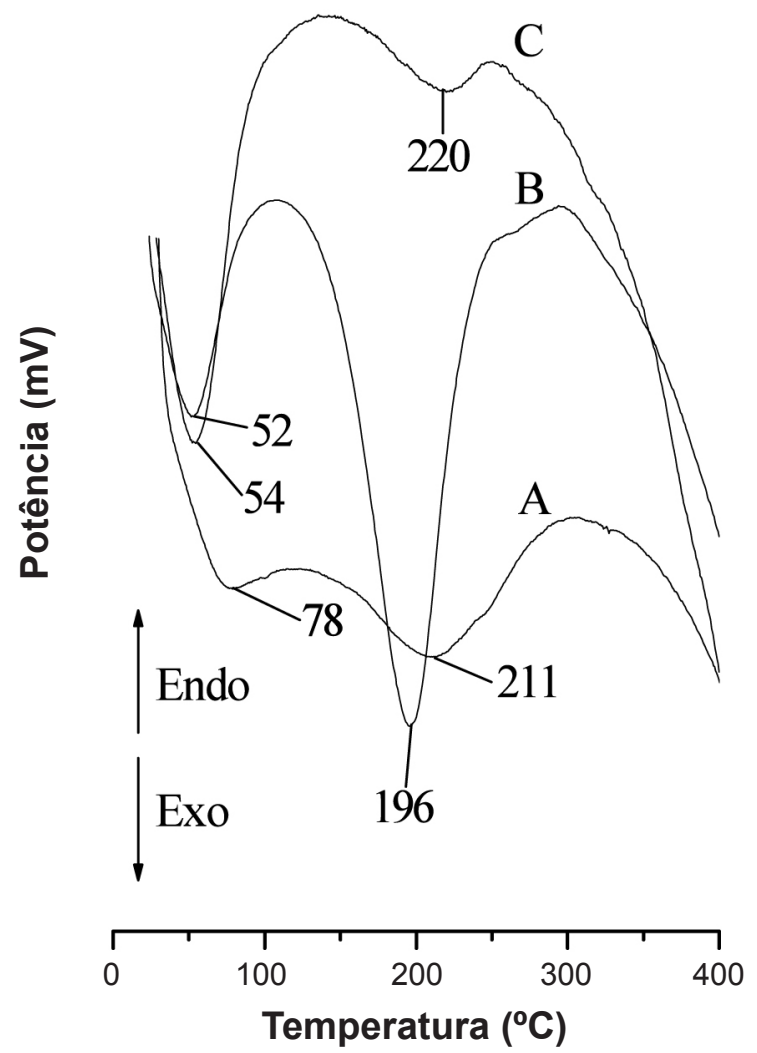

Figura 6: Calorimetria exploratória diferencial das formas modificadas de caulim cinza, com razão de aquecimento de $10^{\circ} \mathrm{C} /$ min em atmosfera de nitrogênio: A) ácido/ácido cítrico; B) ácido/ DMSO e D) ácido/ácido oxálico.

[Figure 6: Curves of differential scanning calorimetry of modified forms of gray kaolin, with a heating rate of $10^{\circ} \mathrm{C} /$ min under $N_{2}: A$ ) acid / citric acid, B) acid / DMSO and D) acid / oxalic acid.

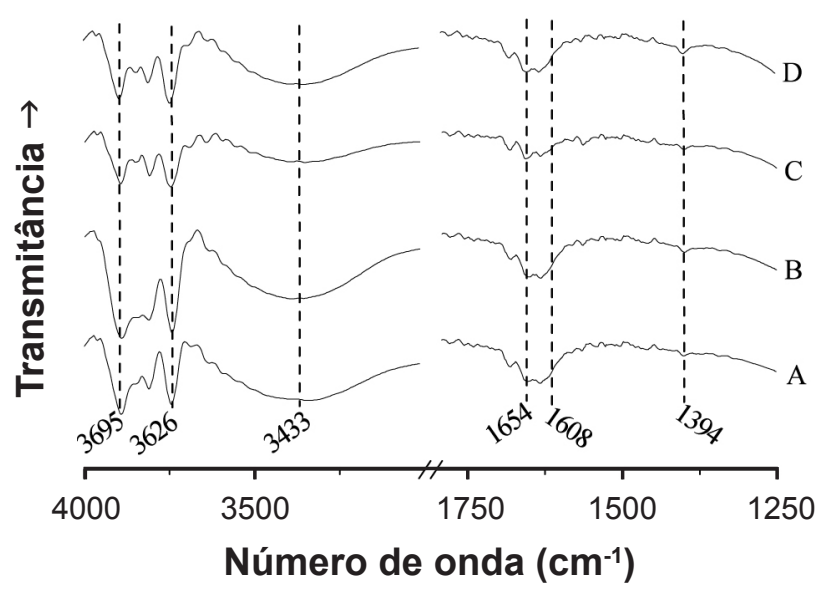

Figura 7: Espectros de absorção na região do infravermelho para: A) caulim branco / natural; B) caulim branco / ácido; C) caulim cinza / natural e D) caulim cinza / ácido.

[Figure 7: Absorption spectra in the infrared region for: A) white kaolin / natural, B) white kaolin / acid, C) gray kaolin/natural and D) grey kaolin / acid.]

Na Fig. 7 são mostrados os espectros do caulim branco natural e da forma ácida. Na região do espectro que compreende as absorções de água características da caulinita, em 3695 a $3100 \mathrm{~cm}^{-1}$, referentes a estiramentos 


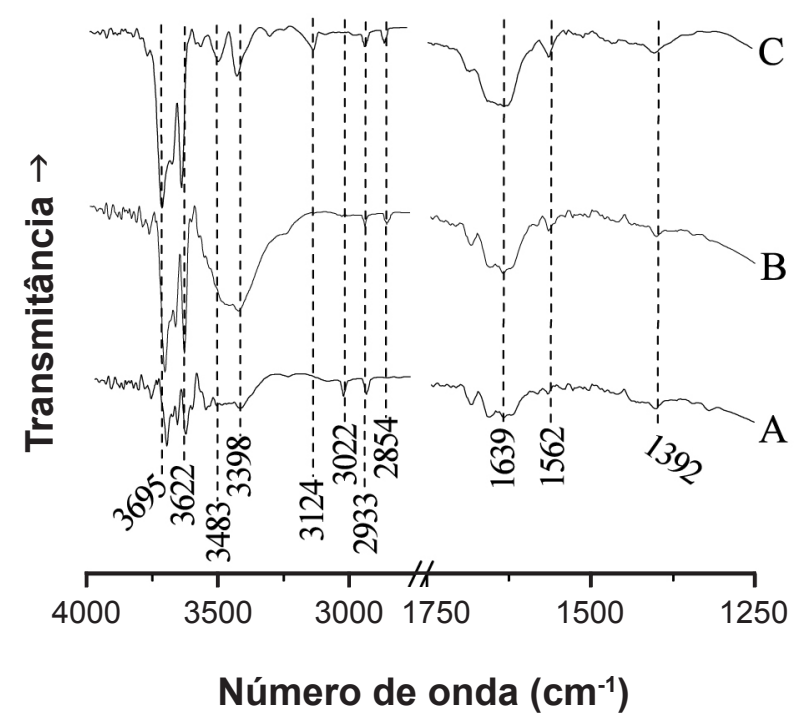

Figura 8: Espectros de absorção na região do infravermelho para o caulim branco: A) ácido/DMSO; B) ácido/ácido oxálico e C) ácido/ ácido cítrico.

[Figure 8: Absorption spectra in the infrared region to the white kaolin: A) acid ( DMSO; B) acid/oxalic acid and C) acid / citric acid.]

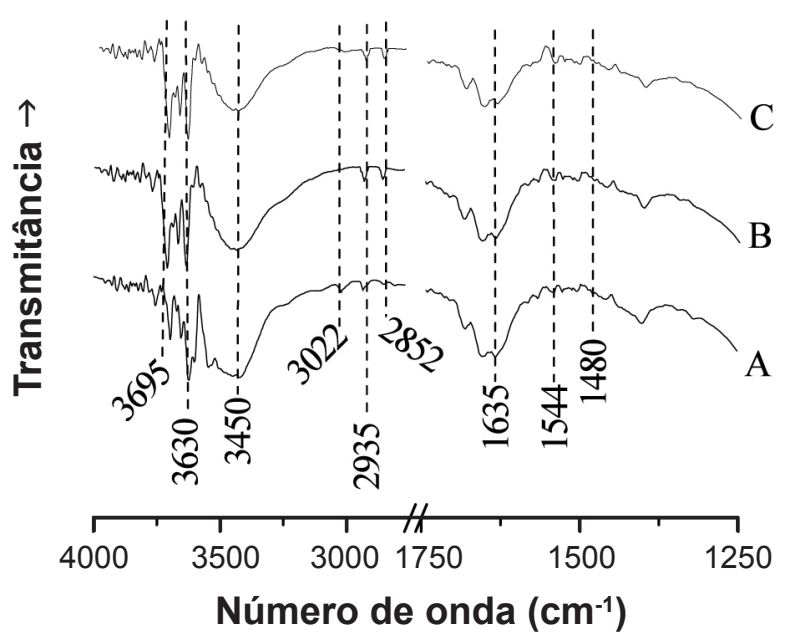

Figura 9: Espectros de absorção na região do infravermelho para o caulim cinza: A) ácido/DMSO, B) ácido/ácido oxálico e C) ácido/ ácido cítrico.

[Figure 9: Absorption spectra in the infrared region to the gray kaolin: A) acid / DMSO, B) acid / oxalic acid and C) acid/citric acid.]

$\mathrm{OH}$, a banda em $3429 \mathrm{~cm}^{-1}$, que está relacionada com ligações de hidrogênio $\mathrm{H}-\mathrm{OH}$ [12]. Nesta mesma região do espectro ocorre a banda em $3626 \mathrm{~cm}^{-1}$ relacionada às hidroxilas octaédricas internas, que também apresentam deformações 1654 e $1608 \mathrm{~cm}^{-1}$, devidas a fortes interações $\mathrm{OH}$ na estrutura.

No espectro A da Fig. 8 ocorre uma banda em 3695 $\mathrm{cm}^{-1}$, que está relacionada à interação do tipo ligação de hidrogênio entre os grupos hidroxila $(\mathrm{OH})$ e os oxigênios dos grupos sulfanila $(\mathrm{S}=\mathrm{O})$ do DMSO $[13,14]$.
Para as amostras modificadas com ácido oxálico e ácido cítrico ocorrem bandas em 2854, 2933, 3022 e $3124 \mathrm{~cm}^{-1}$ nos espectros A, B e C das Figs. 8 e 9, são referentes a estiramento C-H. Já as bandas em 3398 e $3483 \mathrm{~cm}^{-1}$ (Fig. $8 \mathrm{C})$, indicando uma possível ausência de interação entre a caulinita e o ácido cítrico.

\section{CONCLUSÕES}

Não foi detectada matéria orgânica no caulim branco, indicando ser o melhor material precursor em procedimentos de modificação por intercalação, enquanto que o cinza apresenta uma quantidade considerável de matéria orgânica, que segundo vários autores, interfere na capacidade de adsorção da argila, por isso foi submetida a tratamento para remoção. Os resultados das análises de difração de raios $\mathrm{X}$ confirmam uma melhor interação com o DMSO, sendo evidenciado pelo aumento do espaçamento interplanar da estrutura lamelar da caulinita em ambos os caulins. O mesmo não ocorreu com o ácido oxálico e o ácido cítrico. $\mathrm{O}$ grau de intercalação com DMSO foi melhor evidenciado nos difratogramas da amostra de caulim branco na forma ácida. As análises térmicas mostraram resultados que reforçam as considerações sobre a difração de raios X, podendo-se concluir que o caulim branco/DMSO apresentou maiores variações de massa nas análises termogravimétricas e variações de energia mais relevantes nas análises de calorimetria exploratória diferencial. A espectroscopia na região do infravermelho confirma a interação de grupos sulfonila do DMSO presentes na estrutura da caulinita.

\section{AGRADECIMENTOS}

Ao CNPq, à CAPES e à FUNCAP pelo auxílio financeiro.

\section{REFERÊNCIAS}

[1] K. G. Bhattacharyya, S. S. Gupta, Adsorption of a few heavy metals on natural and modified kaolinite and montmorillonite: A review, Adv. Colloid Interface Sci. 140, 2 (2008) 114-131.

[2] P. Ptácek, D. Kubátová, J. Havlica, J. Brandstetr, F. Soukal, T. Opravil, Isothermal J.kinetic analysis of the thermal decomposition of kaolinite: The thermogravimetric study, Thermochim. Acta 501, 1-2 (2010) 24-29.

[3] T. A. Elbokl, C. Detellier, Intercalation of cyclic imides in kaolinite, J. Colloid Interface Sci. 323, 2 (2008) 338-348.

[4] D. L. Guerra, C. Airoldi, K. S. Sousa, Adsorption and thermodynamic studies of $\mathrm{Cu}(\mathrm{II})$ and $\mathrm{Zn}(\mathrm{II})$ on organofunctionalized-kaolinite, Appl. Surface Sci. 254, 16 (2008) 5157-5163.

[5] K. G. Bhattacharyya, S. S. Gupta, Kaolinite and montmorillonite as adsorbents for Fe(III), Co(II) and Ni(II) in aqueous medium, Appl. Clay Sci. 41, 1-2 (2008) 1-9.

[6] C. Volzone, J. Ortiga, Removal of gases by thermalacid leached kaolinitic clays: Influence of mineralogical composition, Appl. Clay Sci. 32 (2006) 87-93. 
[7] T. Megyes, I. Bakó, T. Radnai, T. Grósz, T. Kosztolányi, B. Mroz, M. Probst, Structural investigation of lithium iodide in liquid dimethyl sulfoxide: Comparison between experiment and computation, Chem. Phys. 321 (2006) 100110.

[8] A. R. Fiorucci, M. H. F. B. Soares, E. T. G. Cavalheiro, Ácidos Orgânicos: dos primórdios da Química Experimental à sua presença em nosso cotidiano, Química Nova na Escola (2002) 15.

[9] J. Larsen, C. Cornett, J. W. Jaroszewski, S. H. Hansen, Reaction between drug substances and pharmaceutical excipients: Formation of citric acid esters and amides of carvedilol in the solid state, J. Pharmac. Biomed. Anal. 49 (2009) 11-17.

[10] P. C. Lopes, F. A. Dias, L. R. D. Silva, Decomposition kinetics by thermogravimetry for the intercalation of kaolin with dimethylsulphoxide, Mater. Lett. 57, 22-23 (2003) 3397-3401.

[11] G. W. Brindley, G. Brow, Crystal Structures of Clay Minerals and their X-Ray Identification, Mineralogical Soc., London, Inglaterra (1984) 28-35.

[12] J. M. Hunt, M. P. Whisherd, L. C. Bonham, Analytical Chem, 22, 12 (1950) 1478.

[13] R. K. Vempati, M. Y. A. Mollah, G. R. Reddy, D. L. Cocke, H. V. Lauer Jr., Intercalation of kaolinite under hidrothermal conditions, J. Mater. Sci. 31 (1996) 1255-1259. [14] M. V. S. Fernandes, Avaliação da estabilidade termodinâmica de dimetilsulfóxido intercalado nas estruturas de caulins do nordeste brasileiro, Diss. Mestrado, Depto. Química Orgânica e Inorgânica, Universidade Federal do Ceará - UFC (2007) p. 62-68.

(Rec. 14/12/2009, Rev. 20/06/2010, Ac. 23/07/2010) 